\title{
Recording the self-assembly behavior of nanomaterials directed by hydrogen bonding
}

Ganghuo Pan ${ }^{1 *}$, Jie Leng ${ }^{2}$, Liye Deng ${ }^{3}$, Liwen Xing ${ }^{4}$, Rui Feng ${ }^{5 *}$

${ }^{1}$ School of Chemistry, Beihang University. Beijing 100191, China

${ }^{2}$ Hospital (T.C.M.) Affiliated To SouthWest Medical University, Luzhou, China

${ }^{3}$ Laboratory for Molecular Sciences, CAS Research/Education Center for Excellence in Molecular Sciences, Institute of Chemistry, Chinese Academy of Sciences, Beijing, China

${ }^{4}$ Department of Materials Science and Engineering, College of Chemistry and Materials Engineering, Beijing Technology and Business University, Beijing 100048, China

${ }^{5}$ Beijing National Laboratory for Molecular Sciences (BNLMS), Key Laboratory of Bioorganic Chemistry and Molecular Engineering of Ministry of Education, College of Chemistry, Peking University, Beijing 100871, China

*E-mail: www.p-g-h@163.com 


\section{Experimental section}

\section{Materials and Equipment}

All reagents were directly obtained from commercial suppliers and were analytical grade without further purification. All manipulations were carried out in the laboratory atmosphere. Single crystal structure determination was performed on a Bruker APEXII area detector device with Mo KR radiation $(\lambda=0.71073 \AA)$ by the $\Phi-\omega$ scan method. The transmission electron microscopy (TEM) experiments were recorded on a Hitachi 7650 electron microscope. The scanning electron microscopy (SEM) experiments were recorded on a Hitachi SU8010 electron microscope. The IR spectra were recorded with $\mathrm{KBr}$ pellets on a Bruker EQUINOX $55 \mathrm{FT}$-spectrometer in the range of $4000-400 \mathrm{~cm}^{-1}$. X-ray photoelectron spectroscopy (XPS) was characterized using a Thermo Fisher ESCALAB 250XiPHI Quantera instrument equipped with an Al X-ray excitation source (1486.6 $\mathrm{eV}$ ). Binding energies were corrected by reference to the $\mathrm{C} 1 \mathrm{~s}$ peak at $284.8 \mathrm{eV}$. Elemental analysis (EA) were measured using FlashEA 1112.

\section{S2}

\section{Figures and Tables}
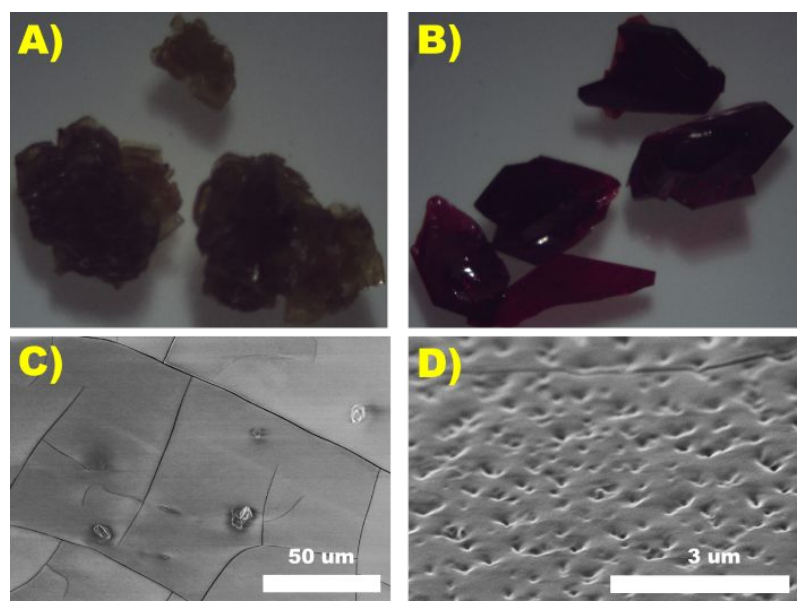

Figure S1. The morphology of single crystal 1 (A) and 2 (B) under optical microscopy. The SEM images of the surface of single crystal 1 (C) and 2(D). 


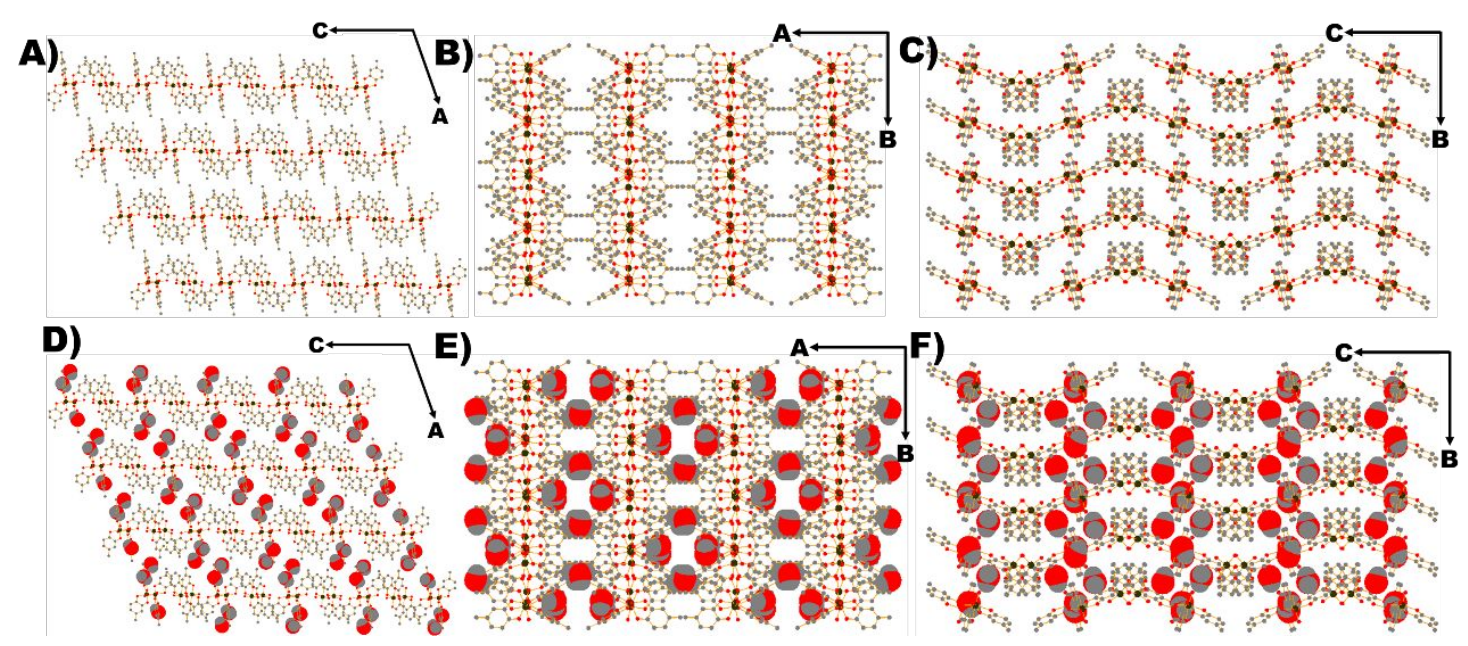

Figure S2. The packing of complex anion $\left[\mathrm{WO}_{2}\left(\mathrm{C}_{7} \mathrm{H}_{6} \mathrm{O}_{2}\right)_{2}\right]^{2-}$ in $a c(\mathrm{~A}), \mathrm{a} b(\mathrm{~B})$ and $\mathrm{b} c(\mathrm{C})$ planes of single crystal 1 , and the packing of complex anion $\left[\mathrm{WO}_{2}\left(\mathrm{C}_{7} \mathrm{H}_{6} \mathrm{O}_{2}\right)_{2}\right]^{2-}$ in $a c$, $\mathrm{a} b$ and $\mathrm{b} c$ planes where $\mathrm{H}_{2} \mathrm{O}$ were filled in the holes of single crystal 2. For clarify, the $\mathrm{H}_{2} \mathrm{O}$ molecules was shown in spacefilling model, the other part such as $\left(\mathrm{NH}_{3} \mathrm{CH}_{2} \mathrm{CH}_{2} \mathrm{CH}_{2} \mathrm{NH}_{2}\right)^{+}$and $\left(\mathrm{NH}_{2} \mathrm{CH}_{2} \mathrm{CH}_{2} \mathrm{CH}_{2} \mathrm{NH}_{2}\right)$ were omitted, and the $\mathrm{H}$ atoms are omitted for clarify except the $\mathrm{H}$ atoms linked with $\mathrm{H}_{2} \mathrm{O}$.

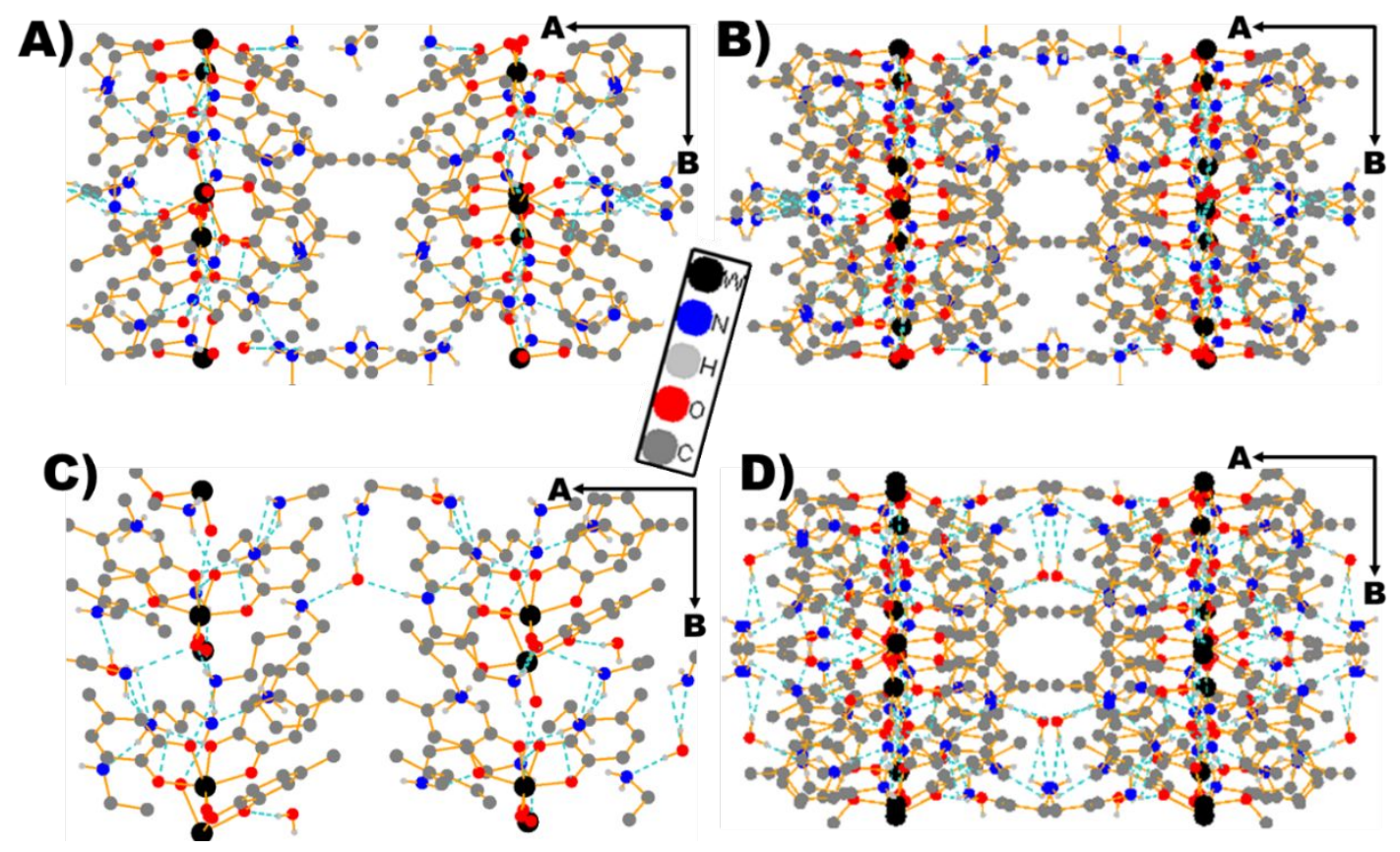

Figure S3. The packing of complex molecules in ab plane of single crystal $1(\mathrm{~A}, \mathrm{~B})$ and 2 (C, D). Ball-and-stick representation of the $1 \mathrm{D}$ quantum wire motifs formed by hydrogen bonds among complex anion $\left[\mathrm{WO}_{2}\left(\mathrm{C}_{7} \mathrm{H}_{6} \mathrm{O}_{2}\right)_{2}\right]^{2-}$ and $\left(\mathrm{NH}_{3} \mathrm{CH}_{2} \mathrm{CH}_{2} \mathrm{CH}_{2} \mathrm{NH}_{2}\right)^{+}$along the direction of $\mathrm{b}$ axis and the 1D quantum wire motifs was linked by $\mathrm{H}_{2} \mathrm{O}$ through $\mathrm{N}-\mathrm{H}$...... $\mathrm{O}$ and $\mathrm{O}-\mathrm{H} \ldots \ldots . \mathrm{N}$ along a axis for single crystal 2. non-bonded hydrogen atoms have been omitted for clarity. 


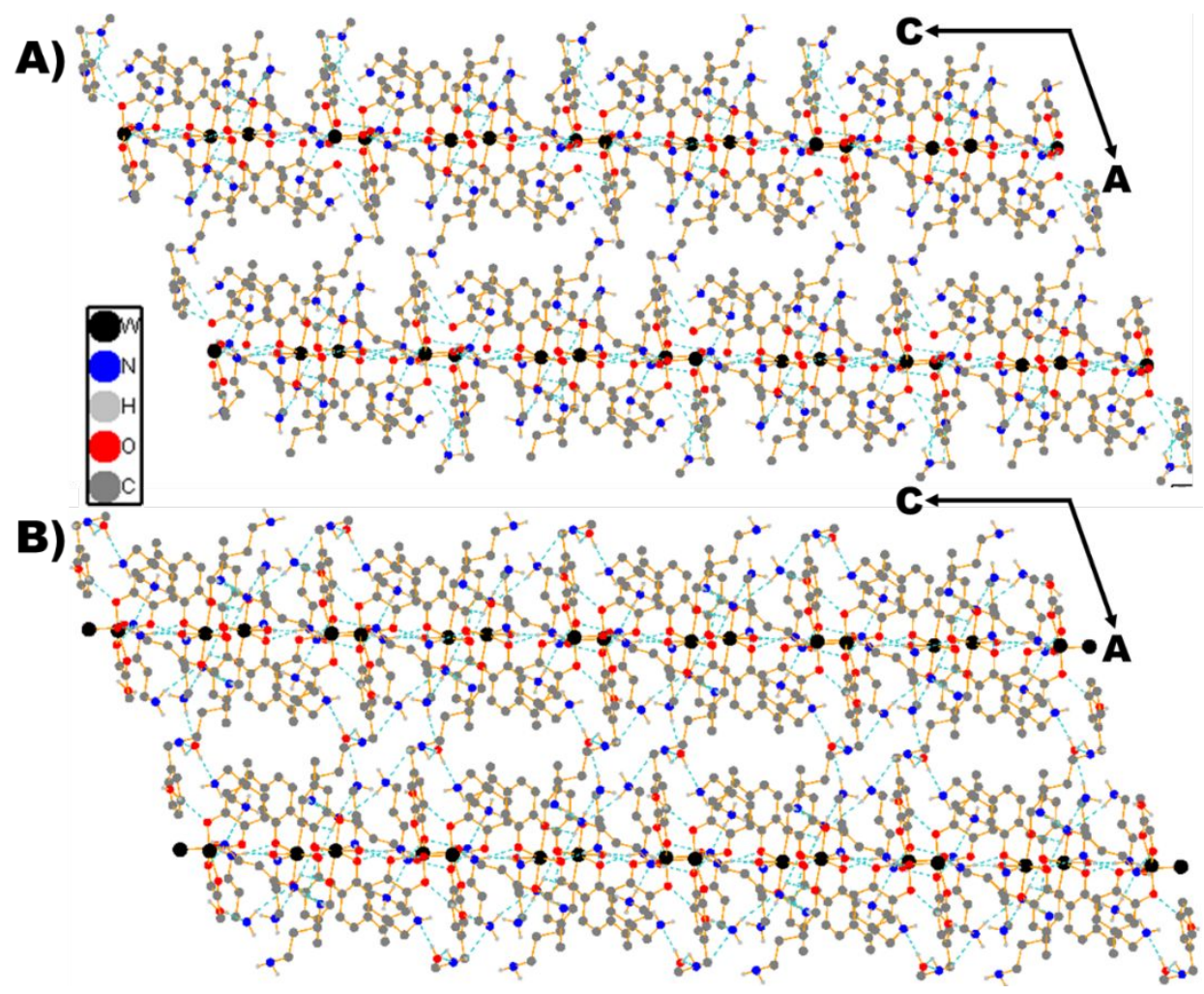

Figure S4. The packing of complex molecules in ac plane of single crystal 1(A, B) and 2 (C, D). Ball-and-stick representation of the 1D quantum wire motifs formed by hydrogen bonds among complex anion $\left[\mathrm{WO}_{2}\left(\mathrm{C}_{7} \mathrm{H}_{6} \mathrm{O}_{2}\right)_{2}\right]^{2-}$ and $\left(\mathrm{NH}_{3} \mathrm{CH}_{2} \mathrm{CH}_{2} \mathrm{CH}_{2} \mathrm{NH}_{2}\right)^{+}$along the direction of $\mathrm{c}$ axis and the 1D quantum wire motifs was linked by $\mathrm{H}_{2} \mathrm{O}$ through $\mathrm{N}-\mathrm{H}$..... $\mathrm{O}$ and $\mathrm{O}-\mathrm{H} . \ldots . . \mathrm{N}$ along a axis for single crystal 2. non-bonded hydrogen atoms have been omitted for clarity.

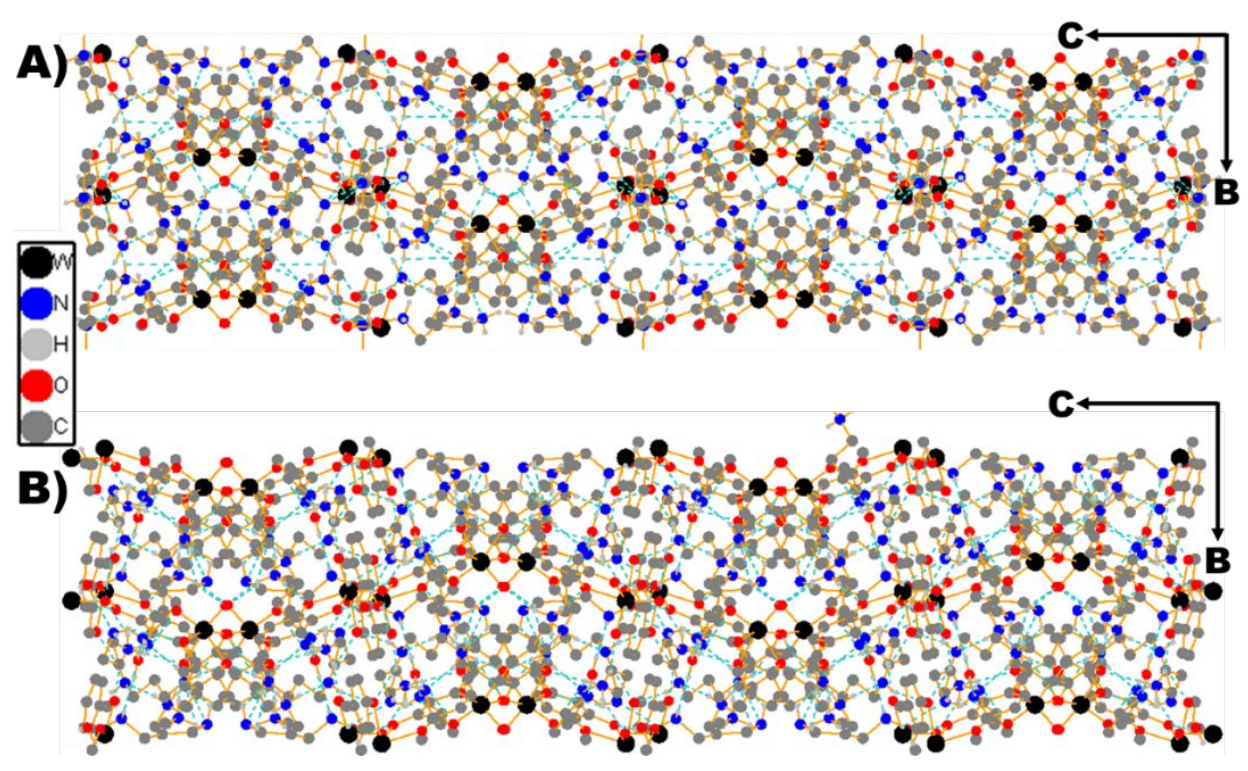

Figure S5. The packing of complex molecules in bc plane of single crystal 1(A, B) and 2 (C, D). Ball-and-stick representation of the $2 \mathrm{D}$ quantum sheet motifs formed by hydrogen bonds among complex anion $\left[\mathrm{WO}_{2}\left(\mathrm{C}_{7} \mathrm{H}_{6} \mathrm{O}_{2}\right)_{2}\right]^{2-}$ and $\left(\mathrm{NH}_{3} \mathrm{CH}_{2} \mathrm{CH}_{2} \mathrm{CH}_{2} \mathrm{NH}_{2}\right)^{+}$in bc plane. non-bonded hydrogen atoms have been omitted for clarity. 


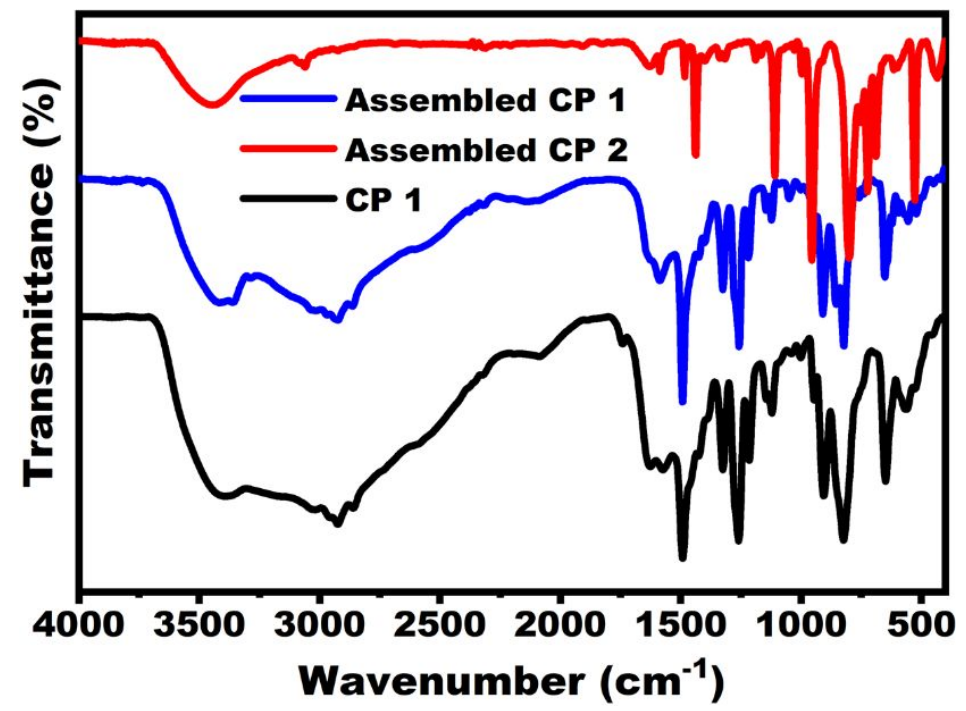

Figure S6. The IR spectra figure of the CP 1, assembled CP1 and assembled CP 2.

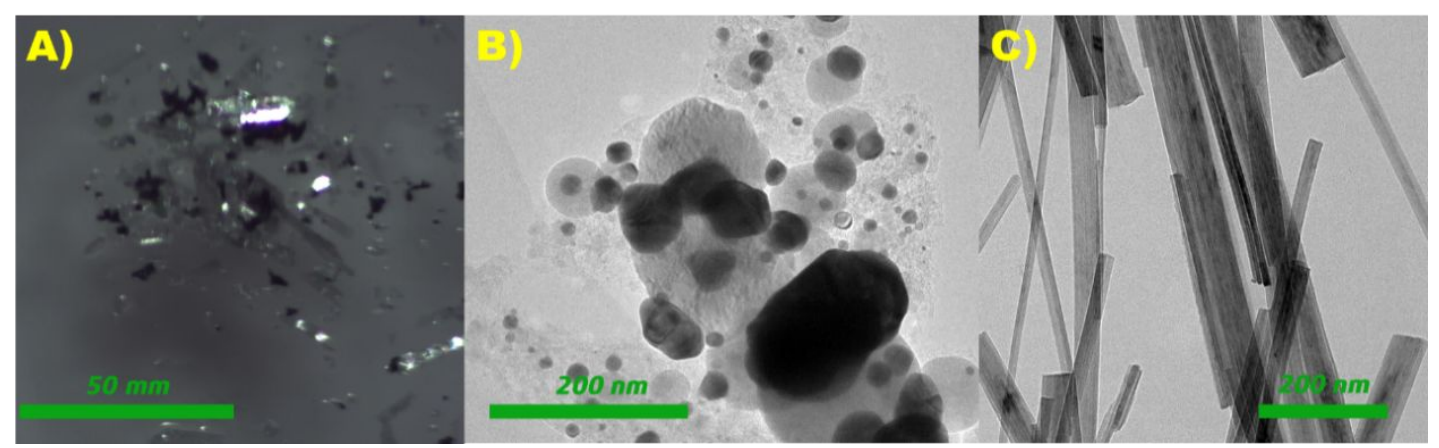

Figure S7. Under optical microscopy, the morphology of $\mathrm{WO}_{3}$ film synthesized from assembled CP2 (A), TEM images of the morphology of $\mathrm{WO}_{3}$ synthesized from block single crystal 2 and assembled $\mathbf{C P 1}$ after heating at $700^{\circ} \mathrm{C}$.

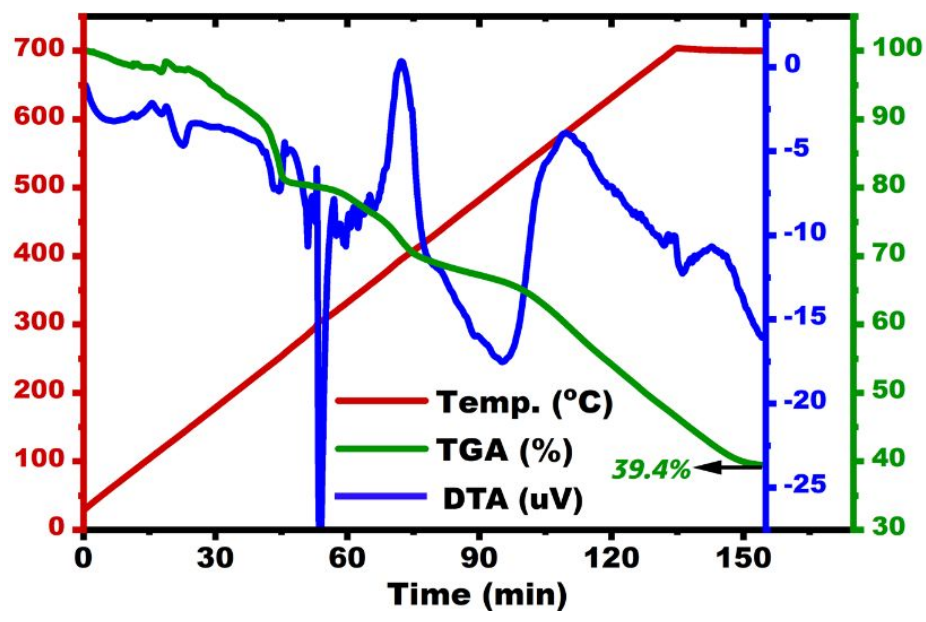

Figure S8. Differential thermograph patterns of assembled CP2. The thermographs were performed in the temperature range $25-700{ }^{\circ} \mathrm{C}$ in nitrogen (Gas Flow: $20 \mathrm{ml} / \mathrm{min}$ ) with a temperature rate 10 o/min. 
We got a kind of ultra-thin $2 \mathrm{D} \mathrm{WO}_{3}$ nanofilm when heated the assembled $\mathbf{C P 2}$ to $700{ }^{\circ} \mathrm{C}$ in nitrogen. We speculate that its formation process was as follow: during the heating process, the structure of assembled CP2 was re-assembled again. When the temperature was heated to about $150{ }^{\circ} \mathrm{C}$, the hydrogen bond formed by water molecules was destroyed and the water molecules were volatilized which was consistent with the weight-loss of TGA (weight loss of $1.48 \mathrm{wt} \%$ calculated and $2.23 \mathrm{wt} \%$ experimental for the volatilization of part 1,3-propanediamine) and thermic changed of DTA (Figure S8). And then the energy provided by heating caused them to undergo secondary self-assembly, forming an intermediate like the intermediate I-VII (ultra-thin nanosheets) of CP1. The ultra-thin nanosheets grew to large area and uncurled without addition force. And finally, these ultra-thin nanofilm decomposed slowly from $300{ }^{\circ} \mathrm{C}$ to $700{ }^{\circ} \mathrm{C}$, and the slow decomposed process at high temperature allowed the decomposed species complete the assembly process and formed the ultrathin $2 \mathrm{D} \mathrm{WO}_{3}$ nanofilm as shown in figure $\mathrm{S} 7$.

Table S1. Crystal data and structure refinement for single crystals 1 and 2

\begin{tabular}{|c|c|c|}
\hline crystal & 1 & 2 \\
\hline Empirical formula & C43H78W2N10O12 & $\mathrm{C} 43 \mathrm{H} 82 \mathrm{~N} 10 \mathrm{O} 14 \mathrm{~W} 2$ \\
\hline Formula mass & 1294.85 & 1330.89 \\
\hline Calcd density, $\mathrm{Mg} / \mathrm{m} 3$ & 1.551 & 1.598 \\
\hline Crystal system & Monoclinic & Monoclinic \\
\hline $\mathrm{a} / \AA$ & $29.102(3)$ & $29.072(3)$ \\
\hline $\mathrm{b} / \AA$ & $13.9465(12)$ & $14.0120(13)$ \\
\hline $\mathrm{c} / \AA$ & $29.779(3)$ & $29.606(3)$ \\
\hline$\alpha /{ }^{\circ}$ & 90 & 90 \\
\hline$\beta /{ }^{\circ}$ & $113.461(3)$ & $113.470(2)$ \\
\hline$\gamma /{ }^{\circ}$ & 90 & 90 \\
\hline Vol, $\AA 3$ & $11087.2(17)$ & $11062.6(17)$ \\
\hline Temperature (K) & $298(2)$ & $298(2)$ \\
\hline space group & $\mathrm{C} 2 / \mathrm{c}$ & $\mathrm{C} 2 / \mathrm{c}$ \\
\hline $\mathrm{Z}$ & 8 & 8 \\
\hline Radiation type & MoK $\backslash \mathrm{a}$ & MoK $\backslash \mathrm{a}$ \\
\hline Absorption coefficient, $\mu / \mathrm{mm}-1$ & 4.209 & 4.223 \\
\hline No. of reflections measured & 27121 & 27635 \\
\hline No. of independent reflections & 9774 & 9745 \\
\hline Rint & 0.1078 & 0.1075 \\
\hline $\mathrm{F}(000)$ & 5200 & 5360 \\
\hline Final R1 values $(\mathrm{I}>2 \sigma(\mathrm{I}))$ & 0.0657 & 0.0823 \\
\hline Final wR(F2) values $(\mathrm{I}>2 \sigma(\mathrm{I}))$ & 0.1683 & 0.2200 \\
\hline Final R1 values (all data) & 0.1446 & 0.1498 \\
\hline Final wR(F2) values (all data) & 0.1965 & 0.2434 \\
\hline Goodness of fit on F2 & 1.048 & 1.109 \\
\hline completeness & $99.8 \%$ & $99.8 \%$ \\
\hline
\end{tabular}


Table S2. Hydrogen bonds for single crystal 1

\begin{tabular}{|llllll}
\hline D-H & $\mathrm{d}(\mathrm{D}-\mathrm{H})$ & $\mathrm{d}(\mathrm{H} . \mathrm{A})$ & $<$ DHA & $\mathrm{d}(\mathrm{D} . . \mathrm{A})$ & $\mathrm{A}$ \\
\hline N1-H1A & 0.890 & 2.108 & 160.49 & 2.961 & $\mathrm{~N} 4[\mathrm{x},-\mathrm{y}+1, \mathrm{z}+1 / 2]$ \\
\hline N1-H1B & 0.890 & 1.981 & 161.83 & 2.840 & $\mathrm{~N} 9[-\mathrm{x}+1 / 2, \mathrm{y}+1 / 2,-\mathrm{z}+3 / 2]$ \\
\hline N1-H1C & 0.890 & 2.009 & 158.34 & 2.856 & $\mathrm{O} 1$ \\
\hline N2-H2B & 0.890 & 2.025 & 155.67 & 2.859 & $\mathrm{O} 7[-\mathrm{x}+1 / 2, \mathrm{y}+1 / 2,-\mathrm{z}+3 / 2]$ \\
\hline N2-H2B & 0.890 & 2.535 & 129.82 & 3.179 & $\mathrm{O} 11[-\mathrm{x}+1 / 2, \mathrm{y}+1 / 2,-\mathrm{z}+3 / 2]$ \\
\hline N2-H2C & 0.890 & 2.127 & 172.79 & 3.012 & $\mathrm{O} 7[\mathrm{x},-\mathrm{y}+1, \mathrm{z}+1 / 2]$ \\
\hline N3-H3A & 0.890 & 1.935 & 169.11 & 2.814 & $\mathrm{O} 5[\mathrm{x},-\mathrm{y}+1, \mathrm{z}-1 / 2]$ \\
\hline N3-H3B & 0.890 & 1.977 & 166.62 & 2.850 & $\mathrm{O} 4[-\mathrm{x}+1 / 2,-\mathrm{y}+3 / 2,-\mathrm{z}+1]$ \\
\hline N3-H3C & 0.890 & 1.897 & 168.94 & 2.776 & $\mathrm{O} 9$ \\
\hline N5-H5B & 0.890 & 1.896 & 165.68 & 2.767 & $\mathrm{~N} 2[-\mathrm{x}+1 / 2, \mathrm{y}-1 / 2,-\mathrm{z}+3 / 2]$ \\
\hline N7-H7A & 0.890 & 2.154 & 137.70 & 2.875 & $\mathrm{O} 4$ \\
\hline N7-H7A & 0.890 & 2.356 & 149.11 & 3.153 & $\mathrm{O} 6$ \\
\hline N7-H7B & 0.890 & 1.957 & 159.17 & 2.806 & $\mathrm{O} 12[-\mathrm{x}+1 / 2,-\mathrm{y}+3 / 2,-\mathrm{z}+1]$ \\
\hline N7-H7C & 0.890 & 1.950 & 167.50 & 2.825 & $\mathrm{O} 11$ \\
\hline N9-H9C & 0.890 & 2.415 & 139.88 & 3.149 & $\mathrm{O} 5$ \\
\hline N10-H10C & 0.890 & 2.336 & 116.40 & 2.845 & $\mathrm{~N} 8[-\mathrm{x},-\mathrm{y}+1,-\mathrm{z}+1]$ \\
\hline
\end{tabular}

Table S3. Hydrogen bonds for single crystal 2

\begin{tabular}{|lcllll}
\hline D-H & $d(D-H)$ & $d(H . . A)$ & $<$ DHA & $d(D . . A)$ & $A$ \\
\hline N1-H1A & 0.890 & 2.034 & 161.03 & 2.890 & $\mathrm{~N} 10$ \\
\hline N1-H1B & 0.890 & 1.998 & 163.22 & 2.861 & $\mathrm{~N} 7[-\mathrm{x}+3 / 2, \mathrm{y}+1 / 2,-\mathrm{z}+1 / 2]$ \\
\hline N1-H1C & 0.890 & 1.983 & 176.23 & 2.872 & $\mathrm{O} 3$ \\
\hline N2-H2B & 0.890 & 1.992 & 163.03 & 2.855 & $\mathrm{O} 7[-\mathrm{x}+3 / 2, \mathrm{y}+1 / 2,-\mathrm{z}+1 / 2]$ \\
\hline N2-H2B & 0.890 & 2.569 & 124.67 & 3.161 & $\mathrm{O} 11[-\mathrm{x}+3 / 2, \mathrm{y}+1 / 2,-\mathrm{z}+1 / 2]$ \\
\hline N2-H2C & 0.890 & 2.085 & 168.17 & 2.962 & $\mathrm{O} 7[\mathrm{x},-\mathrm{y}+1, \mathrm{z}-1 / 2]$ \\
\hline N3-H3A & 0.890 & 1.972 & 170.55 & 2.853 & $\mathrm{O} 2[-\mathrm{x}+3 / 2,-\mathrm{y}+3 / 2,-\mathrm{z}+1]$ \\
\hline N3-H3B & 0.890 & 2.033 & 139.73 & 2.773 & $\mathrm{O} 11[-\mathrm{x}+3 / 2,-\mathrm{y}+3 / 2,-\mathrm{z}+1]$ \\
\hline N3-H3C & 0.890 & 1.948 & 158.63 & 2.795 & $\mathrm{O} 12$ \\
\hline N5-H5B & 0.890 & 1.984 & 168.36 & 2.861 & $\mathrm{~N} 2[\mathrm{x},-\mathrm{y}+2, \mathrm{z}+1 / 2]$ \\
\hline N7-H7B & 0.890 & 2.418 & 111.05 & 2.861 & $\mathrm{~N} 1[-\mathrm{x}+3 / 2, \mathrm{y}-1 / 2,-\mathrm{z}+1 / 2]$ \\
\hline N9-H9A & 0.890 & 1.895 & 168.60 & 2.773 & $\mathrm{O} 9[\mathrm{x},-\mathrm{y}+1, \mathrm{z}-1 / 2]$ \\
\hline N9-H9B & 0.890 & 1.993 & 162.98 & 2.856 & $\mathrm{O} 2[-\mathrm{x}+3 / 2, \mathrm{y}-1 / 2,-\mathrm{z}+1 / 2]$ \\
\hline N9-H9C & 0.890 & 1.982 & 164.44 & 2.850 & $\mathrm{O} 5$ \\
\hline N10-H10B & 0.890 & 2.186 & 170.11 & 3.067 & $\mathrm{O} 14[\mathrm{x},-\mathrm{y}+1, \mathrm{z}-1 / 2]$ \\
\hline O13-H13C & 0.850 & 2.211 & 167.99 & 3.048 & $\mathrm{O} 10$ \\
\hline O13-H13D & 0.850 & 2.126 & 166.80 & 2.960 & $\mathrm{~N} 4[-\mathrm{x}+3 / 2,-\mathrm{y}+3 / 2,-\mathrm{z}+1]$ \\
\hline O14-H14F & 0.850 & 1.977 & 174.59 & 2.824 & $\mathrm{~N} 6[\mathrm{x}-1 / 2, \mathrm{y}-1 / 2, \mathrm{z}]$ \\
\hline O14-H14G & 0.850 & 2.222 & 162.32 & 3.043 & $\mathrm{~N} 8[-\mathrm{x}+3 / 2,-\mathrm{y}+1 / 2,-\mathrm{z}+1]$ \\
\hline
\end{tabular}

